\title{
The potential scarcity, or not, of polymeric overall water splitting
}

\section{photocatalysts}

Benedict Saunders, ${ }^{1,2}$ Liam Wilbraham, ${ }^{1,3}$ Andrew W. Prentice, ${ }^{1}$ Reiner Sebastian Sprick, ${ }^{4}$ Martijn A. Zwijnenburg ${ }^{1, *}$

${ }^{1}$ Department of Chemistry, University College London, 20 Gordon Street, London WC1H OAJ, U.K.

${ }^{2}$ Currently at the Department of Chemistry, University of Warwick, Coventry CV4 7SH, U.K.

${ }^{3}$ Currently at Exscientia, Oxford Science Park, Oxford, OX4 4GE, U.K.

${ }^{4}$ Department of Pure and Applied Chemistry, University of Strathclyde, 295 Cathedral Street, Glasgow, G1 1XL, U.K.

"Email: m.zwijnenburg@ucl.ac.uk

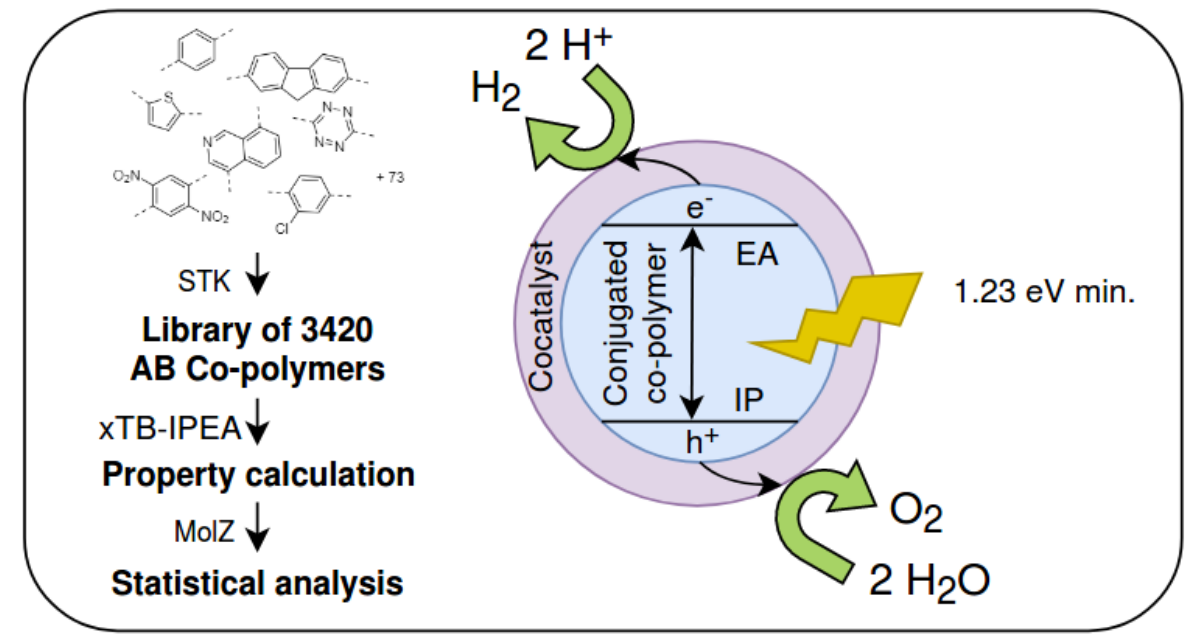

We perform a high-throughput virtual screening of a set of 3240 conjugated alternating binary co-polymers and homo-polymers, in which we predict their ability to drive sacrificial hydrogen evolution and overall water splitting when illuminated with visible light. We use the outcome of this screening to analyse how common the ability to drive either reaction is for conjugated polymers loaded with suitable co-catalysts, and to suggest promising (co-)monomers for polymeric overall water splitting catalysts. 


\section{Introduction}

In recent years organic materials, particularly conjugated polymers, have come to the foreground as potential photocatalysts for splitting water into molecular hydrogen and oxygen. ${ }^{1-5}$ The hydrogen produced in this way would be renewable and green, emitting nothing but water upon combustion, making it a viable candidate for a sustainable replacement for current carbonaceous fuels. While more than a hundred polymers and other organic materials are known to be experimentally active for photocatalytic hydrogen evolution when studied in the presence of a metal co-catalyst and a sacrificial electron donor (SED), ${ }^{2,6-7}$ a much smaller number has been observed to oxidise water in the presence of a co-catalyst and a sacrificial electron acceptor (SEA), ${ }^{8-11}$ and a smaller number still with the ability to drive overall water splitting when illuminated by solar light. ${ }^{2,12-16}$ Examples of the latter include carbon nitride ${ }^{12-13,16}$ and covalent triazine-based framework materials. ${ }^{14-15}$ Here, we computationally screen a very large dataset of organic (co-)polymers to understand if this apparent experimental scarcity of organic materials that, under illumination with visible light, can oxidise water and drive the overall splitting of water is due to thermodynamic constraints inherent to the material class or is kinetic in nature. In the latter case materials that appear inactive could potentially become active in the presence of the right co-catalyst(s) or when part of a heterojunction, accelerating otherwise sluggish water oxidation kinetics and/or minimising undesired electron-hole recombination.

From a thermodynamic perspective, for a material to reduce protons and oxidise water when illuminated, the material's ionisation potential (IP, valence band maximum) and electron affinity (EA, conduction band minimum) should at least straddle the solution proton reduction and water oxidation potentials, the latter defined - as is convention - in terms of the potential of the equivalent reduction reaction (see figure 1a). Similarly, to reduce protons and oxidise a SED or oxidise water and reduce a SEA the material's IP and EA should straddle the proton reduction and SED oxidation or the SEA reduction and water oxidation potentials, respectively, once again defining the oxidation potentials in terms of that of the equivalent reduction half-reactions. It is highly desirable not only for the material to be active, but to be active under visible light, as most of the sunlight is concentrated in this part of the spectrum. Therefore, 
the material's optical gap, the energy below and wavelength above which the material is transparent to light, needs to be as small as possible. However, at the same time the materials' optical gap needs to be larger than the difference between the potentials of the relevant solution reduction and oxidation half-reactions, 1.23 (e)V in the case of overall water splitting. Otherwise, the photons would not deliver enough energy for the relevant overall reaction to be thermodynamically favourable. The fact that the exciton binding energy in organic materials, even after immersion in water, is not negligible and that the excitons, excited electron-hole pairs, formed initially by the absorption of light do not spontaneously dissociate into free electrons and holes complicates the above picture slightly. ${ }^{17}$ Conceptually, in the case of materials with a non-negligible exciton binding energy we need to consider besides IP and EA also IP* and EA*, the ionisation potential and electron affinity of the excitons, respectively. ${ }^{18-19}$ However, in practice when screening materials the effect of the missing exciton binding energy, typically $0.1-0.2$ (e) $\mathrm{V}^{17}$ can be absorbed in a requirement for a similar overpotential on top of the thermodynamic solution potentials.

Calculating the IP, EA, and optical gap of thousands of polymers with (timedependent) density functional theory ((TD-)DFT) would be prohibitively computationally expensive. However, we demonstrated previously that calculations using the xTB family of tight-binding DFT methods,$^{20}$ developed by Grimme and coworkers, after calibration to (TD-)DFT results give very similar results for a fraction of the computational $\cos ^{21}$ and allow for the convenient calculation of the properties of tens of thousands molecules and/or polymers. ${ }^{6,22-24}$ The xTB calculations, GFN1-xTB ${ }^{25}$ for ground-state geometries, IPEA-xTB ${ }^{26}$ for IP and EA, and STDA-xTB ${ }^{27}$ for the optical gap, are performed on a single polymer chain, embedded in the case of GFN1-xTB and IPEA-xTB in a dielectric continuum with the relative dielectric permittivity of water. In previous work, it was shown that use of such a minimal model of a polymer in DFT calculation gives a good fit to experimentally measured IP and EA values for dry polymer solids. ${ }^{28-29}$ Using calibrated XTB we predict the IP, EA and optical gap values of a dataset consisting of 3240 conjugated alternating binary co-polymers and homopolymers built from a library of 80 monomers, see figures $1 \mathrm{~b}$ and $1 \mathrm{c}$, and analyse what fraction of the dataset can evolve hydrogen in the presence of a commonly used SED, 
triethylamine (TEA), and what fraction of polymers can drive the overall splitting of water. We discuss how these numbers change when we require the polymer to work using visible light and, finally, present the monomers which are overrepresented in the polymers that are active in the visible. Such monomers would make good candidates to explore experimentally when synthesising polymer photocatalysts.

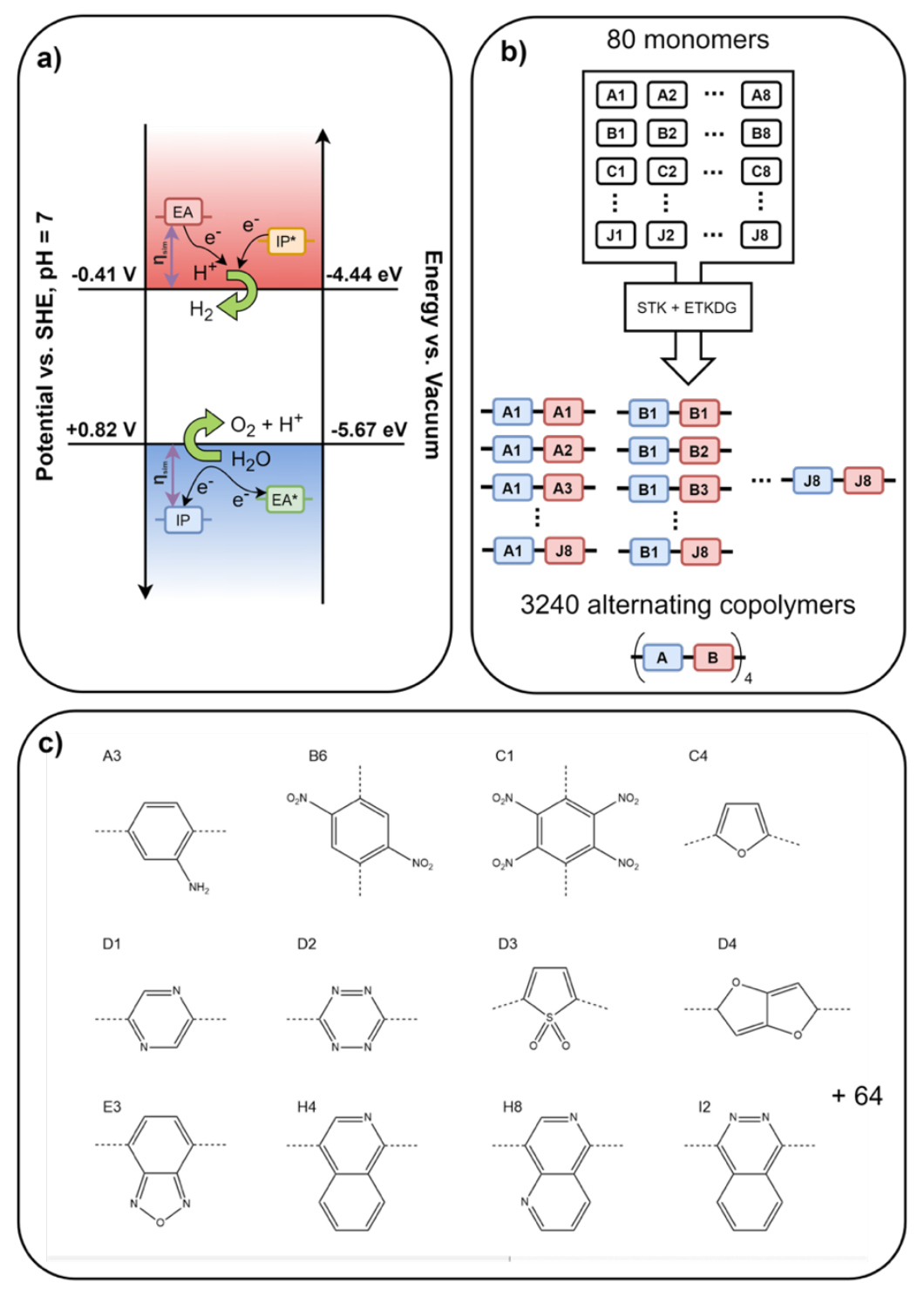

Fig. 1 (a) Schematic illustration of the alignment of IP and EA, and their excited state counterparts IP* and $E A^{*}$, relative to the potentials for proton reduction and water oxidation required for overall water splitting, (b) Oligomer assembly methodology, (c) Subset of the 80 monomers studied herein (all 80 monomers are shown in figure S1 in the supplementary information). 


\section{Methodology}

We assembled 3240 oligomers from all possible binary AB combinations of the library of 80 monomers, including 80 homo-oligomers (see figures 1b). A selection of the monomers is shown in figure $1 \mathrm{C}$; the full library is displayed in figure $\mathrm{S} 1$ in the supporting information. For each oligomer, a SMILES representation was generated using the $\mathrm{stk}^{30-31}$ python package, such that each oligomer contained 16 individual monomers (8 -AB- repeat units for the alternating co-polymers, 16 -A-repeat units for the homo-polymers). As SMILES strings contain no explicit geometry information, a subsequent conformer search was required which we performed using the ETKDG ${ }^{32}$ algorithm as implemented in $R D K_{i t}{ }^{33}$ a cheminformatics library, generating 500 conformers for each oligomer and optimising each with the MMFF94 ${ }^{34}$ forcefield. The conformer with the lowest forcefield energy was selected and optimised further with GFN1-xTB,$^{25}$ as implemented in xtb 5.6.4SE, ${ }^{35}$ in preparation for the calculation of IP, EA and optical gap.

The IPEA-xTB ${ }^{25}$ variant of xTB was used to predict the vertical IP and EA values of the oligomer, with solvation in water represented by the generalised Born surface area (GBSA) model. ${ }^{36}$ The singlet excitation spectra of the oligomers were calculated with the sTDA-xTB ${ }^{27}$ method, the lowest of which was taken to be the optical gap. The sTDAxTB calculations do not include solvation as that is not implemented for sTDA-xTB. The GFN1/IPEA/sTDA-xTB predicted IP, EA and optical gap values for each oligomer were then transformed to values that can be directly compared to previous (TD-)DFT predictions through a linear scaling model, calibrated in prior work by $\mathrm{us}^{21}$ to (TD-)DFT results for oligomers immersed in water. An independent linear model was used for each of the three properties, see Table S1 for the model parameters used. The mean average deviation (MAD) of these models based on the initial training set were 0.08 , 0.06 and 0.13 (e)V for IP/EA/optical gap respectively, increasing to 0.16/0.14/0.16 (e)V for a validation set of polymers not included in the training set. These MAD values are reasonable such that the calibration should be transferrable to the wide range of polymers considered herein.

A locally adapted version of the MolZ python package ${ }^{37}$ allows us to query our dataset and assign a z-score to each of the 80 monomers, which provides an insight into the 
contribution of each monomer to the queried properties. To calculate the z-score we describe the statistics of the scenario where we have a dataset of $N$ polymers, $k$ of which contain a particular monomer $i$, and $n$ polymers in a sub-set of the dataset with desirable properties, $p$ of which contain $i$, in terms of a hypergeometric distribution with a mean $(\bar{x})$ and variance $\left(\sigma^{2}\right)$ of:

$$
\begin{gathered}
\bar{x}=\frac{n k}{N} \\
\sigma^{2}=\frac{n k(N-k)(N-n)}{N^{2}(N-1) .}
\end{gathered}
$$

From the mean and variance of the distribution we can then calculate the z-score for a particular value of $p$ via:

$$
\text { z-score }_{i}=\frac{(p-\bar{x})}{\sigma^{2} .}
$$

Large positive z-scores indicate that monomer $i$ is overrepresented in the sub-set of polymers relative to other monomers and hence likely a good monomer choice for achieving the properties representative of that subset.

\section{Results and Discussion}

First, we analyse what fraction of the 3240 polymers can evolve hydrogen in the presence of TEA as the SED. As can be seen from figure 2a more than $95 \%$ of the polymers in the dataset, even if limiting ourselves to polymers with an absorption onset in the visible region (figure $2 \mathrm{~b}$ ), can thermodynamically drive the reduction of protons and the 2-electron oxidation of TEA to diethylamine and acetaldehyde at $\mathrm{pH} 12.3$ $(-0.76 \mathrm{~V})$. The latter is the experimentally determined $\mathrm{pH}$ of the mixture of TEA, water and methanol used in photocatalysis experiments. ${ }^{38}$ The percentage of polymers able to drive both half-reactions decreases steadily when we require an additional overpotential for both of the two half-reactions. Still, for an overpotential value of 0.3 $\mathrm{V}$, proton reduction and 2-electron oxidation of TEA is predicted to be favourable for more than $80 \%$ of the dataset.

Proton reduction and hydrogen-hydrogen bond formation is typically catalysed by added platinum nanoparticles or palladium nanoparticles remaining from the catalyst used in polymer synthesis. ${ }^{39-40}$ Polymers can also potentially act as a catalyst for proton 
reduction themselves but in the presence of palladium or platinum nanoparticles the activity of those will very likely outcompete any inherent catalytic activity of the polymer. ${ }^{41}$ In contrast, TEA oxidation most likely is not catalytic, and probably takes the form of two sequential out-of-sphere electron-transfer steps between the polymer and TEA. First TEA gets oxidised to TEA ${ }^{+}$, which after deprotonation, gets oxidised in the second electron-transfer step to diethylamine and acetaldehyde, the expected 2electron oxidation products. To efficiently oxidise TEA a polymer's IP should thus probably not only be more positive than the potential of the half-reaction associated with the 2-electron oxidation of TEA but also be more positive than that for the 1electron oxidation of TEA ( $0.64 \mathrm{~V}$ at $\mathrm{pH} 12.3)$, as otherwise the 1-electron oxidation step might act as a thermodynamic barrier. ${ }^{42}$ Indeed, truly active polymers for hydrogen evolution in the presence of TEA generally have such positive IP values. ${ }^{21}$ Going back to figure $2 \mathrm{a}$ we can see that a smaller percentage of the dataset can drive both proton reduction and the 1-electron oxidation of TEA at $\mathrm{pH} 12.3$, between 60 and $70 \%$ in the absence of an overpotential requirement and approximately $40 \%$ for an overpotential of $0.3 \mathrm{~V}$. Figures $2 \mathrm{c}$ and $2 \mathrm{~d}$ finally demonstrate that if we require a polymer not only be able to reduce protons and oxidise TEA but also to have an optical gap that is small enough to absorb a large part of the visible spectrum the percentages get truly small. This reduction, especially in the case when considering the 2-electron oxidation of TEA and proton reduction, is mainly the direct results of the optical gap restriction. Of the 3240 polymers in the dataset 3197 (98.7\%) have an optical gap below $3.5 \mathrm{eV}, 1184$ (36.5\%) have an optical gap below $2.5 \mathrm{eV}$ and only 267 (8.2\%) have an optical gap below $2.0 \mathrm{eV}$ (see dashed lines in figures $2 \mathrm{~b}-2 \mathrm{~d}$ ). However, when considering the 1electron oxidation of TEA and proton reduction there is a clear further reduction, which probably stems from the fact that polymers with a small optical gap also will have a small fundamental gap, the difference between IP and EA, which might straddle the potentials for the 2-electron oxidation of TEA and proton reduction but not those for the 1-electron oxidation of TEA and proton reduction. 
a)

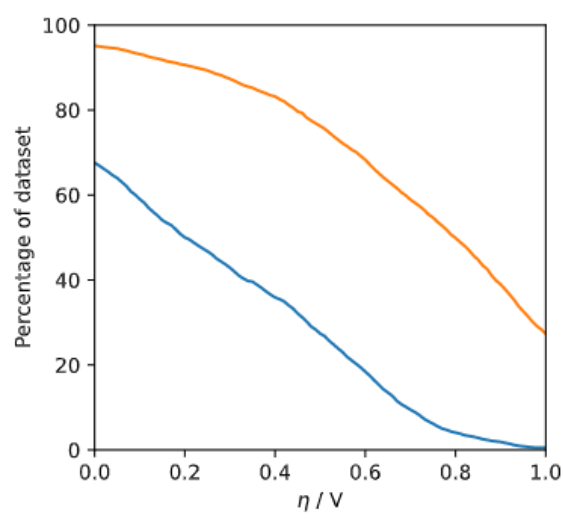

c)

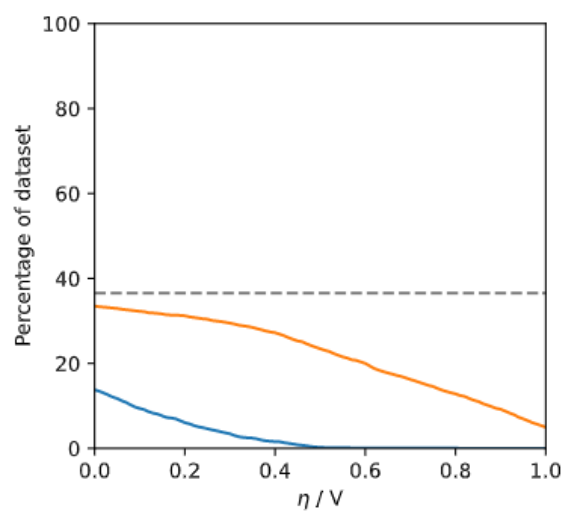

b)

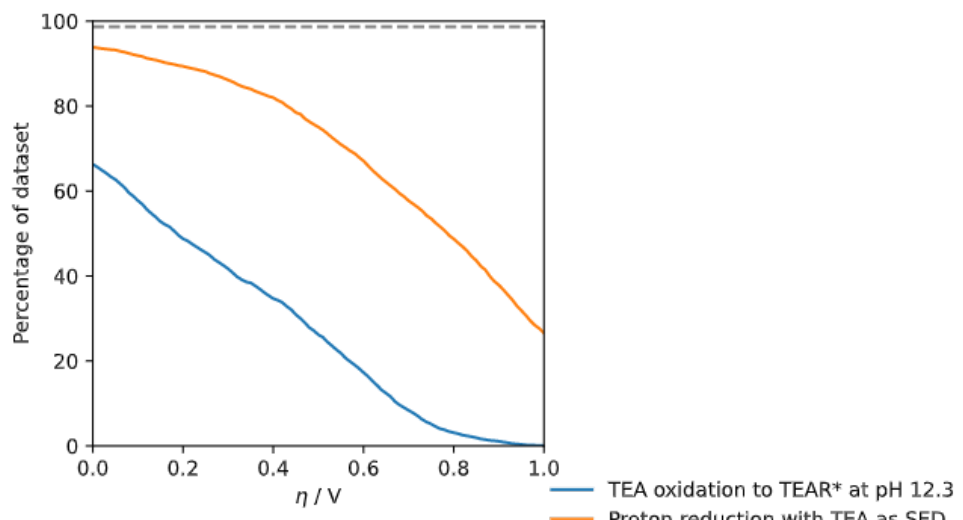

d)

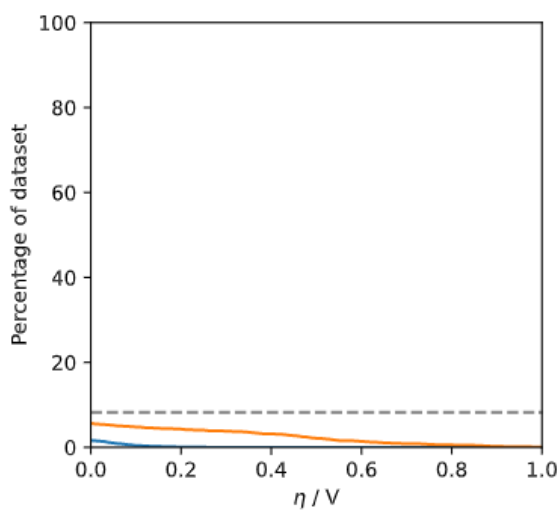

Fig. 2 Percentage of (co-)polymers in the dataset that are predicted to be able to drive the reduction of protons and the overall oxidation of TEA (orange line) and the one-hole oxidation of TEA (blue line) at pH 12.3 for different optical gap ranges. Panel (a) does not include a limitation on the optical gap. Panel (b) introduces a limitation such that the predicted optical gap must be $\leq 3.5 \mathrm{eV}$, which is reduced to $2.5 \mathrm{eV}$ in plot (c) and finally $2.0 \mathrm{eV}$ in plot (d). The grey dashed lines in panels $b$-d indicate the number of polymers in the entire dataset that fall within each optical gap constraint. See figure S2 for a zoomed in version of $a-d$.

Figure 3 shows the case of overall water splitting but now as a function of $\mathrm{pH}$. For neutral water, $\mathrm{pH} 7$, more than $50 \%$ of polymers are predicted to straddle the proton reduction and water oxidation potentials, which reduces to a still respectable more than $30 \%$ when requiring an additional $0.3 \mathrm{~V}$ overpotential for both half-reactions. The fraction of polymers able to both reduce protons and oxidise water, for each overpotential value, increase steadily with $\mathrm{pH}$. At $\mathrm{pH} 12.3$, the same $\mathrm{pH}$ as for when using TEA as SED, we predict that approximately $70 \%$ of polymers straddle the proton reduction and water oxidation potentials and when requiring an additional $0.3 \mathrm{~V}$ 
overpotential still more than $40 \%$ do so. These numbers are surprisingly similar to those discussed above for the case of where TEA instead of water is used as electron donor. Also, similar to when TEA is used as the SED, figures $3 \mathrm{c}$ and $3 \mathrm{~d}$ show that if we require additionally for the polymer to have an optical gap small enough to absorb a large part of the visible spectrum relatively few polymers remain. Of the 3240 polymers in the dataset 3141 (96.9\%) have an optical gap between 1.23 and $3.5 \mathrm{eV}, 1128$ (34.8\%) have an optical gap between 1.23 and $2.5 \mathrm{eV}$ and only 211 (6.5\%) have an optical gap below 2 but above $1.23 \mathrm{eV}$ (see dashed lines in figures 3b-3d). Moreover, just as for the case of the 1-electron oxidation of TEA and proton reduction, discussed above, only a fraction of those polymers with an optical gap between 1.23 and $2.5 \mathrm{eV}$ and 1.23 and $2.0 \mathrm{eV}$ will straddle the water splitting potentials. Even if all polymers with an optical gap between 1.23 and $2.5 \mathrm{eV}$ or 1.23 and $2.0 \mathrm{eV}$ would have an optical gap larger than $1.23 \mathrm{~V}$, their IP and EA still might not straddle the water oxidation and proton reduction potentials because their IP is too deep or their EA to shallow. In contrast, polymers with a large optical gap will likely also have large fundamental gaps and thus will span the water splitting potentials for most realistic IP and EA values. 
a)

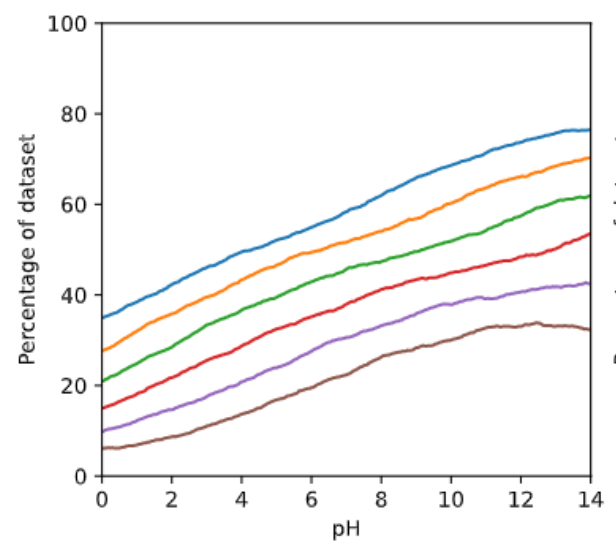

c)

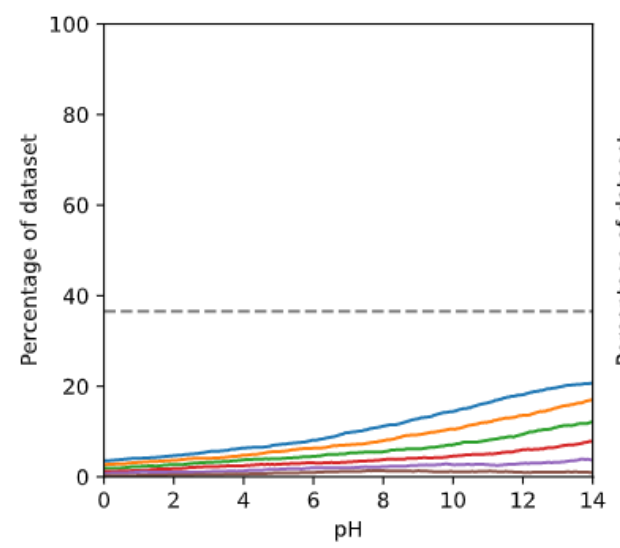

b)

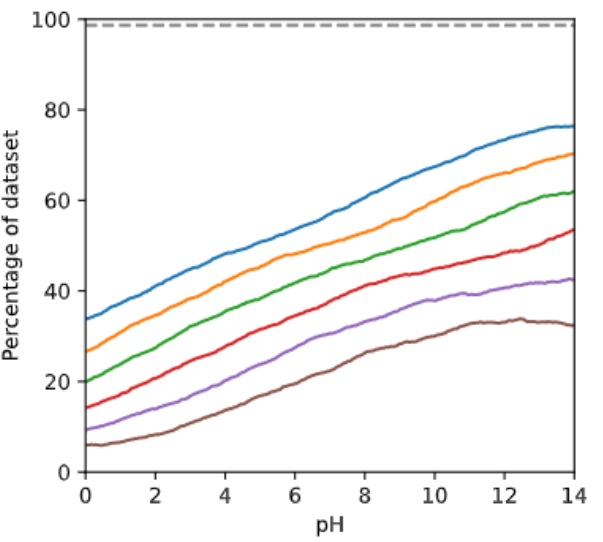

d)

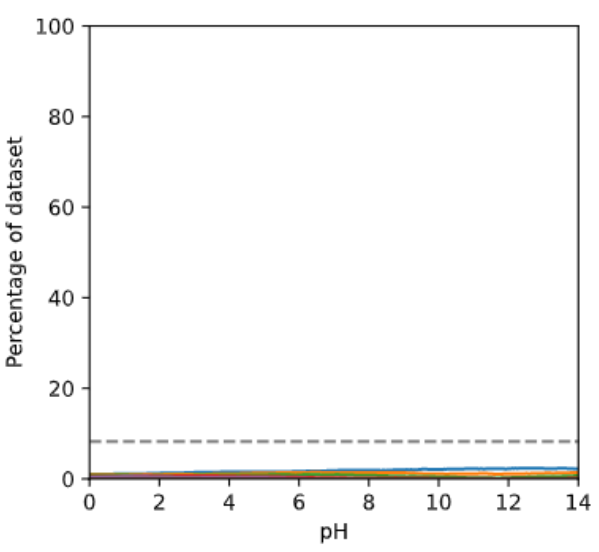

Fig. 3 Percentage of (co-)polymers in the dataset that are predicted to be able to drive the overall splitting of water as a function of $\mathrm{pH}$ for different overpotential values and optical gap ranges. Panel (a) includes all polymers predicted to have an optical gap between 0.0 and $3.5 \mathrm{eV}$, panel (b) those with an optical gap between $1.23 \mathrm{eV}$ and $3.5 \mathrm{eV} \mathrm{f}$, panel (c) those with an optical gap between $1.23 \mathrm{eV}$ and $2.5 \mathrm{eV}$, and $1.23 \mathrm{eV}$ and $2.0 \mathrm{eV}$ for panel (d). The grey dashed lines in panels $b$-d indicate the number of polymers in the entire dataset that fall within each optical gap constraint. See figure S3 for zoomed in version of $a-d$.

The origin of the experimental scarcity of polymers that split water appears thus not to find its origin in any thermodynamic limitations of the material class. Many polymers are known to evolve hydrogen in the presence of TEA and very few to perform overall water splitting, even if in terms of thermodynamics similar numbers of polymers can drive both half-reaction pairs. We hypothesize that the origin of the scarcity of polymer photocatalysts for overall water splitting is kinetic rather than thermodynamic in nature. Overall TEA oxidation involves two electrons (holes) and water oxidation 
four, and while after the first electron transfer step TEA ${ }^{+}$likely diffuses away from the polymer, reducing the chance of the back electron transfer, the water oxidation intermediates probably stay adsorbed on co-catalyst surface until oxygen is formed, making back electron transfer more likely. Non-catalytic water oxidation by means of out-of-sphere electron transfer in analogy to the case of TEA is very unlikely because of the extreme potential of half-reaction associated with the initial one-hole oxidation of water (2.15 V at $\mathrm{pH} 7)$. In the case of a sluggish water-oxidation co-catalyst, electronhole recombination hence likely outcompetes water oxidation. Similarly, sacrificial electron acceptors used in the case of sacrificial water oxidation, e.g., $\mathrm{Ag}^{+}$and $\mathrm{Ce}^{4+}$, generally are thermodynamically easier to reduce than protons, i.e., have a more positive reduction potential, and only involve one rather than two electrons, meaning that in the presence of a sluggish water-oxidation co-catalyst, sacrificial water oxidation might be possible but overall water splitting not. The good news associated with this scenario is that this would mean that by choosing the right water oxidation co-catalyst a significant number of polymers should be active for overall water splitting. Indeed, experimentally the small number of polymers active for overall water splitting, were generally first reported to be only active for sacrificial water oxidation ${ }^{8,10}$ and only after optimisation of the water oxidation co-catalyst became active for overall water splitting. ${ }^{13,15}$ It would also suggest that combining polymers or polymers and small molecule acceptors together to make heterojunctions, splitting the excitons, and separating electrons and holes on separate sub-systems, reducing electron-hole recombination, should be a worthwhile strategy. As a matter of fact, bulk heterojunctions have been shown to yield promising results in the case of sacrificial hydrogen evolution. ${ }^{43}$ Even in the presence of a heterojunction, however, the presence of efficient hydrogen and oxygen evolution co-catalysts is probably beneficial in terms of the long-term stability of the polymer. Stability is not generally an issue in sacrificial hydrogen evolution with continuous hydrogen evolution for multiple days demonstrated for selected polymer materials without obvious signs of material degradation during this time. ${ }^{29,44-45}$ However, the build-up of charges in the polymer, ${ }^{46}$ especially holes, during overall water splitting in the presence of a sluggish water oxidation co-catalyst could result in material degradation and loss of activity. Reversely, a very active water oxidation co-catalyst, which very efficiently extracts 
charges from the polymer, might result in very stable polymer photocatalysts under overall water splitting conditions. For this reason, we also rule out that stability or lack thereof is the/a reason for the scarcity of overall water splitting photocatalysts.

One possible hurdle to efficient overall water splitting with polymer photocatalysts, even in the presence of optimal co-catalysts, is that relatively few (co)polymers are, as discussed above, predicted to have the required driving force for proton reduction and water oxidation and absorb green to red light. However, it is important to stress here that such (co-)polymers are predicted to exist, discussed in more detail below, and this is not an issue unique to polymeric photocatalysts. As a matter of fact, many state-of-the-art inorganic solid-state photocatalysts, such as doped $\mathrm{SrTiO}_{3}$, only absorb blue, violet and ultraviolet light ${ }^{47-48}$ and developing systems that are active on the red-side of the visible spectrum is also a challenge for inorganic materials. ${ }^{47-48}$
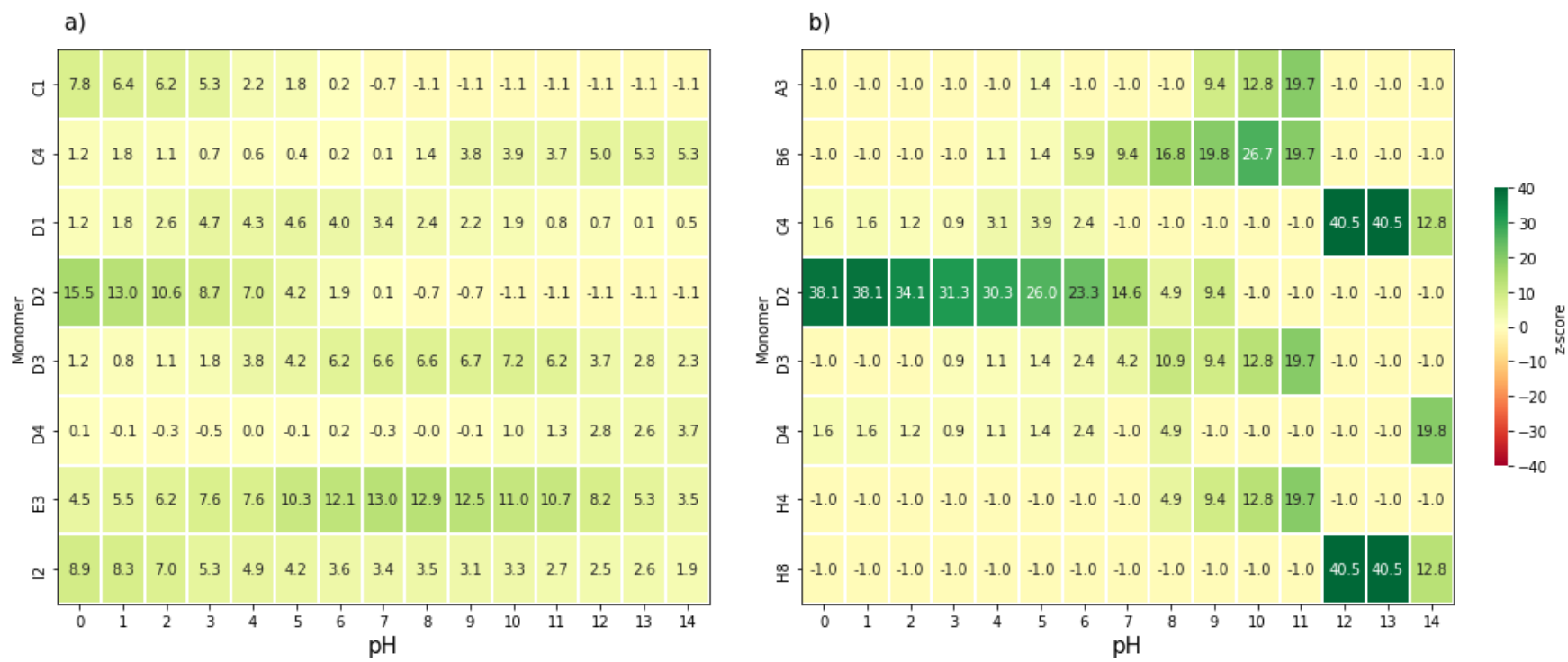

Fig. 4 Heatmaps of the monomers with the highest z-score for watersplitting with overpotentials of $0.3 \mathrm{~V}$, constrained by optical gap ranges (a) $1.23-2.5 \mathrm{eV}$ and (b) 1.23 $-2.0 \mathrm{eV}$. The monomers which feature here correspond to those illustrated in figure $1 \mathrm{c}$.

Finally, we analysed, by means of z-scores, which monomers are overrepresented under those binary $\mathrm{AB}$ co-polymers that can drive overall water splitting and absorb a large part of the visible spectrum. Figures $4 \mathrm{a}$ and $4 \mathrm{~b}$ show the monomers with the highest z-score as a function of $\mathrm{pH}$ for polymers that can drive 
overall water splitting with an overpotential of $0.3 \mathrm{~V}$ and a predicted optical gap between $1.23 \mathrm{eV}$ and $2.5 \mathrm{eV}$ or $2.0 \mathrm{eV}$, respectively. At acidic $\mathrm{pH}$, the monomer with the highest z-score for both optical gap ranges is 3,6-dibromo-1,2,4,5-tetrazine (D2, see figure 1C). This monomer occurs in 15 out of the $16(<2.0 \mathrm{eV})$ and 15 out of the $38(<2.5 \mathrm{eV})$ co-polymers that can drive overall water splitting at $\mathrm{pH} 0$, respectively. For polymers with an optical gap below $2.5 \mathrm{eV}$ several other monomers in the same neutral to acidic $\mathrm{pH}$ range also have relatively high z-scores: 1,4-dibromophthalazine (I2, occurring in 9 of the 38 co-polymers at $\mathrm{pH} 0$ ) and 1,4-dibromo-2,3,5,6tetranitrobenzene ( $\mathrm{C} 1$, occurring in 8 of the 38 co-polymers at $\mathrm{pH} 0$ ), see figure $4 \mathrm{a}$. In contrast, as can be seen in figure 4b, no other monomers than D2 have high z-scores at acidic $\mathrm{pH}$ when we limit the optical gap to $2.0 \mathrm{eV}$. For D2, I2 and $\mathrm{C} 1$ the z-scores increase with decreasing $\mathrm{pH}$, in line with the fact that these are all electron poor monomers that are likely to result in co-polymers with deep, very positive, IP values, and the fact that at low $\mathrm{pH}$ polymers are most likely limited by their ability to oxidise water. At high $\mathrm{pH}$, for polymers with an optical gap below $2.0 \mathrm{eV}, 2,5$-dibromofuran (C4) and 5,8-dibromo-1,6-naphthyridine (H8), as well as 2,5-dibromo-2,5dihydrofuro[3,2-b]furan (D4), have the highest z-scores, see figure $4 \mathrm{~b}$. At these $\mathrm{pH}$ values, $>\mathrm{pH} 12$, water oxidation is thermodynamically easier relative to at low $\mathrm{pH}$, explaining why some of these monomers are relatively electron rich (C4, D4). The overrepresentation of these monomers at alkaline $\mathrm{pH}$ values probably arises from the fact that co-polymers containing them have low(er) optical gaps rather than a deep IP. However, one should be careful not to overinterpret this data as the high z-scores in this region arise in part because very few polymers are predicted to have an optical gap $<2.0 \mathrm{eV}$ and able to drive overall water splitting, specifically 6 polymers at $\mathrm{pH} 14,3$ of which contain $\mathrm{D} 4$ and 2 containing $\mathrm{C} 4$ or $\mathrm{H} 8$. For intermediate $\mathrm{pH}$ values between neutral and alkaline, 4,7-dibromobenzo[c][1,2,5]oxadiazole (E3) has a relatively high z-score for polymers with an optical gap below $2.5 \mathrm{eV}$. E3 occurs in 35 out of $107 \mathrm{co}$ polymers with an optical gap $<2.5 \mathrm{eV}$ that can drive overall water splitting at $\mathrm{pH} 7$.
1,4-Dibromo-2,5-dinitrobenzene
(B6),
2,5-dibromoaniline
(A3), 2,5dibromothiophene-1,1-dioxide (D3) and 1,4-dibromoisoquinoline (H4) have similarly high z-scores for polymers with an optical gap below $2.0 \mathrm{eV}$. Polymers based on all these monomers, other than 1,4-dibromo-2,3,5,6-tetranitrobenzene which is likely to 
be explosive and best avoided for that reason, should make good choices for future synthetic studies towards overall water splitting polymer photocatalysts. This is especially true of monomers like D2 and E3 for which a large number of polymers are predicted to be able to drive overall water splitting and have a small optical gap: 15 out of the 80 (co-)polymers in the data-set containing D2 (19\%) are predicted to have an optical gap $<2.0 \mathrm{eV}$ and able to drive overall water splitting at acid $\mathrm{pH}$ while 35 out the 80 (co-)polymers containing E3 in the data-set (44\%) are predicted to have an optical gap $<2.5 \mathrm{eV}$ and able to drive overall water splitting at neutral $\mathrm{pH}$. Leads in term of co-monomers in the case of $\mathrm{D} 2$ at acidic $\mathrm{pH}$ include 3,7dibromodibenzo[b,d]thiophene-5,5-dioxide (J8), 2,5-dibromopyrazine (D1), 2,5dibromopyrimidine (C8), 2,7-dibromo-9H-fluoren-9-one (J5), 2,5dibromoterephthalonitrile (B2), 1,4-dibromo-2,5-bis(trifluoromethyl)benzene (B7), 1,4-dibromo-2-nitrobenzene (A8) and E3. Similarly, co-monomer leads for E3 at neutral $\mathrm{pH}$ include $\mathrm{A} 8, \mathrm{~B} 2, \mathrm{~B} 7, \mathrm{C} 8, \mathrm{D} 1$, 1,4-dibromo-2-fluorobenzene (A2), 1,4dibromo-2,5-difluorobenzene (A6), 1,4-dibromo-2,3,5,6-tetrafluorobenzene (B4), 2,5dibromobenzonitrile (A4), 1,4-dibromo-2-(trifluoromethyl)benzene (B1), 2,5dibromopyridine (C7) and most nitrogen substituted naphtalenes (e.g. E6 and F2).

\section{Conclusions}

Thermodynamically a significant number of conjugated polymers are predicted to be able to drive overall water splitting, a similar number as polymers able to drive hydrogen evolution when oxidising triethylamine as a sacrificial electron donor. As many polymers are known experimentally to evolve hydrogen in the presence of triethylamine, we argue that the scarcity of polymers performing overall water splitting is not due to any thermodynamic constraints but rather probably has a kinetic origin, with overall water splitting requiring an efficient co-catalyst for the water oxidation. Moreover, in the presence of such co-catalysts, it is likely that a significant number of polymers that are very active for sacrificial hydrogen evolution, will also be active for overall water splitting. Considering the rapid development of polymer photocatalysts and increase in sacrificial hydrogen production rates we believe that now more effort must be made to study water oxidation with polymer photocatalysts, focusing on developing suitable co-catalysts, given that this is currently a bottleneck in developing 
systems for overall water splitting and our work suggests that this is a realistic target. A large hurdle for efficient overall water splitting with visible light, finally, is found to be the fact that only a relatively small number of the co-polymers studied are predicted to absorb yellow to red light. However, this is similarly an issue for inorganic photocatalysts, with many state-of-the-art inorganic photocatalysts, e.g. $\mathrm{SrTiO}_{3}$, only absorbing blue, violet and ultraviolet light.

\section{Acknowledgements}

We thank Dr. Yang Bai, Dr. Isabelle Heath-Apostolopoulos and Prof. Andrew Cooper for discussion and acknowledge the UK Engineering and Physical Sciences Research Council (EPSRC) and the Leverhulme Trust for funding this work through grants EP/N004884/1 and RPG-2019-209, respectively. R.S.S thanks the University of Strathclyde for financial support through The Strathclyde Chancellor's Fellowship Scheme.

\section{References}

1. Vyas, V. S.; Lau, V. W.-h.; Lotsch, B. V., Soft Photocatalysis: Organic Polymers for Solar Fuel Production. Chemistry of Materials 2016, 28, 5191-5204.

2. Wang, Y.; Vogel, A.; Sachs, M.; Sprick, R. S.; Wilbraham, L.; Moniz, S. J. A.; Godin, R.; Zwijnenburg, M. A.; Durrant, J. R.; Cooper, A. I.; Tang, J., Current Understanding and Challenges of Solar-Driven Hydrogen Generation Using Polymeric Photocatalysts. Nature Energy 2019, 4, 746-760.

3. Kosco, J.; Moruzzi, F.; Willner, B.; McCulloch, I., Photocatalysts Based on Organic Semiconductors with Tunable Energy Levels for Solar Fuel Applications. Advanced Energy Materials 2020, 10, 2001935.

4. Banerjee, T.; Podjaski, F.; Kröger, J.; Biswal, B. P.; Lotsch, B. V., Polymer Photocatalysts for Solar-to-Chemical Energy Conversion. Nature Reviews Materials 2021, 6, 168-190.

5. Bai, Y.; Hippalgaonkar, K.; Sprick, R. S., Organic Materials as Photocatalysts for Water Splitting. Journal of Materials Chemistry A 2021, 9, 16222-16232.

6. Bai, Y.; Wilbraham, L.; Slater, B. J.; Zwijnenburg, M. A.; Sprick, R. S.; Cooper, A. I., Accelerated Discovery of Organic Polymer Photocatalysts for Hydrogen Evolution from Water through the Integration of Experiment and Theory. Journal of the American Chemical Society 2019, 141, 9063-9071.

7. Meier, C. B.; Clowes, R.; Berardo, E.; Jelfs, K. E.; Zwijnenburg, M. A.; Sprick, R. S.; Cooper, A. I., Structurally Diverse Covalent Triazine-Based Framework Materials for Photocatalytic Hydrogen Evolution from Water. Chemistry of Materials 2019, 31, 8830-8838. 8. Wang, X.; Maeda, K.; Thomas, A.; Takanabe, K.; Xin, G.; Carlsson, J. M.; Domen, K.; Antonietti, M., A Metal-Free Polymeric Photocatalyst for Hydrogen Production from Water under Visible light. Nature Materials 2009, 8, 76-80. 
9. $\quad B i$, J.; Fang, W.; Li, L.; Wang, J.; Liang, S.; He, Y.; Liu, M.; Wu, L., Covalent TriazineBased Frameworks as Visible Light Photocatalysts for the Splitting of Water. Macromolecular Rapid Communications 2015, 36, 1799-1805.

10. Lan, Z.-A.; Fang, Y.; Zhang, Y.; Wang, X., Photocatalytic Oxygen Evolution from Functional Triazine-Based Polymers with Tunable Band Structures. Angewandte Chemie International Edition 2018, 57, 470-474.

11. Sprick, R. S.; Chen, Z.; Cowan, A. J.; Bai, Y.; Aitchison, C. M.; Fang, Y.; Zwijnenburg, M. A.; Cooper, A. I.; Wang, X., Water Oxidation with Cobalt-Loaded Linear Conjugated Polymer Photocatalysts. Angewandte Chemie International Edition 2020, 59, 18695-18700.

12. Liu, J.; Liu, Y.; Liu, N.; Han, Y.; Zhang, X.; Huang, H.; Lifshitz, Y.; Lee, S.-T.; Zhong, J.; Kang, Z., Metal-Free Efficient Photocatalyst for Stable Visible Water Splitting Via a TwoElectron Pathway. Science 2015, 347, 970-974.

13. Zhang, G.; Lan, Z.-A.; Lin, L.; Lin, S.; Wang, X., Overall Water Splitting by Pt/G-C3N4 Photocatalysts without Using Sacrificial Agents. Chemical Science 2016, 7, 3062-3066.

14. Kong, D.; Han, X.; Xie, J.; Ruan, Q.; Windle, C. D.; Gadipelli, S.; Shen, K.; Bai, Z.; Guo, Z.; Tang, J., Tunable Covalent Triazine-Based Frameworks (CTF-0) for Visible-Light-Driven Hydrogen and Oxygen Generation from Water Splitting. ACS Catalysis 2019, 9, 7697-7707. 15. Zhang, S.; Cheng, G.; Guo, L.; Wang, N.; Tan, B.; Jin, S., Strong-Base-Assisted Synthesis of a Crystalline Covalent Triazine Framework with High Hydrophilicity Via Benzylamine Monomer for Photocatalytic Water Splitting. Angewandte Chemie International Edition 2020, 59, 6007-6014.

16. Pan, Z.; Liu, M.; Zhang, G.; Zhuzhang, H.; Wang, X., Molecular Triazine-Heptazine Junctions Promoting Exciton Dissociation for Overall Water Splitting with Visible Light. The Journal of Physical Chemistry C 2021, 125, 9818-9826.

17. Guiglion, P.; Butchosa, C.; Zwijnenburg, M. A., Polymer Photocatalysts for Water Splitting: Insights from Computational Modeling. Macromolecular Chemistry and Physics 2016, 217, 344-353.

18. Guiglion, P.; Butchosa, C.; Zwijnenburg, M. A., Polymeric Watersplitting Photocatalysts; a Computational Perspective on the Water Oxidation Conundrum. Journal of Materials Chemistry A 2014, 2, 11996-12004.

19. Prentice, A. W.; Zwijnenburg, M. A., The Role of Computational Chemistry in Discovering and Understanding Organic Photocatalysts for Renewable Fuel Synthesis. Advanced Energy Materials 2021, 11, 2100709.

20. Bannwarth, C.; Caldeweyher, E.; Ehlert, S.; Hansen, A.; Pracht, P.; Seibert, J.; Spicher, S.; Grimme, S., Extended Tight-Binding Quantum Chemistry Methods. WIREs Computational Molecular Science 2021, 11, e1493.

21. Wilbraham, L.; Berardo, E.; Turcani, L.; Jelfs, K. E.; Zwijnenburg, M. A., HighThroughput Screening Approach for the Optoelectronic Properties of Conjugated Polymers. Journal of Chemical Information and Modeling 2018, 58, 2450-2459.

22. Heath-Apostolopoulos, I.; Wilbraham, L.; Zwijnenburg, M. A., Computational HighThroughput Screening of Polymeric Photocatalysts: Exploring the Effect of Composition, Sequence Isomerism and Conformational Degrees of Freedom. Faraday Discussions 2019, 215, 98-110.

23. Wilbraham, L.; Smajli, D.; Heath-Apostolopoulos, I.; Zwijnenburg, M. A., Mapping the Optoelectronic Property Space of Small Aromatic Molecules. Communications Chemistry 2020, 3, 14. 
24. Heath-Apostolopoulos, I.; Vargas-Ortiz, D.; Wilbraham, L.; Jelfs, K. E.; Zwijnenburg, M. A., Using High-Throughput Virtual Screening to Explore the Optoelectronic Property Space of Organic Dyes; Finding Diketopyrrolopyrrole Dyes for Dye-Sensitized Water Splitting and Solar Cells. Sustainable Energy \& Fuels 2021, 5, 704-719.

25. Grimme, S.; Bannwarth, C.; Shushkov, P., A Robust and Accurate Tight-Binding Quantum Chemical Method for Structures, Vibrational Frequencies, and Noncovalent Interactions of Large Molecular Systems Parametrized for All SPD-Block Elements ( $Z=1-86$ ). Journal of Chemical Theory and Computation 2017, 13, 1989-2009.

26. Ásgeirsson, V.; Bauer, C. A.; Grimme, S., Quantum Chemical Calculation of Electron Ionization Mass Spectra for General Organic and Inorganic Molecules. Chemical Science 2017, 8, 4879-4895.

27. Grimme, S.; Bannwarth, C., Ultra-Fast Computation of Electronic Spectra for Large Systems by Tight-Binding Based Simplified Tamm-Dancoff Approximation (sTDA-xTB). The Journal of Chemical Physics 2016, 145, 054103.

28. Guiglion, P.; Monti, A.; Zwijnenburg, M. A., Validating a Density Functional Theory Approach for Predicting the Redox Potentials Associated with Charge Carriers and Excitons in Polymeric Photocatalysts. The Journal of Physical Chemistry C 2017, 121, 1498-1506.

29. Woods, D. J.; Hillman, S. A. J.; Pearce, D.; Wilbraham, L.; Flagg, L. Q.; Duffy, W.; McCulloch, I.; Durrant, J. R.; Guilbert, A. A. Y.; Zwijnenburg, M. A.; Sprick, R. S.; Nelson, J.; Cooper, A. I., Side-Chain Tuning in Conjugated Polymer Photocatalysts for Improved Hydrogen Production from Water. Energy \& Environmental Science 2020, 13, 1843-1855.

30. Turcani, L.; Berardo, E.; Jelfs, K. E., STK: A Python Toolkit for Supramolecular Assembly. Journal of Computational Chemistry 2018, 39, 1931-1942.

31. https://github.com/lukasturcani/stk.

32. Riniker, S.; Landrum, G. A., Better Informed Distance Geometry: Using What We Know to Improve Conformation Generation. Journal of Chemical Information and Modeling 2015, 55, 2562-2574.

33. https://www.rdkit.org/.

34. Halgren, T. A., Merck Molecular Force Field. I. Basis, Form, Scope, Parameterization, and Performance of MMFF94. Journal of Computational Chemistry 1996, 17, 490-519.

35. https://github.com/grimme-lab/xtb.

36. Onufriev, A. V.; Case, D. A., Generalized Born Implicit Solvent Models for Biomolecules. Annual Review of Biophysics 2019, 48, 275-296.

37. https://github.com/LiamWilbraham/molz.

38. Bai, Y.; Wilbraham, L.; Gao, H.; Clowes, R.; Yang, H.; Zwijnenburg, M. A.; Cooper, A. I.; Sprick, R. S., Photocatalytic Polymers of Intrinsic Microporosity for Hydrogen Production from Water. Journal of Materials Chemistry A 2021, 9, 19958-19964.

39. Kosco, J.; Sachs, M.; Godin, R.; Kirkus, M.; Francas, L.; Bidwell, M.; Qureshi, M.; Anjum, D.; Durrant, J. R.; McCulloch, I., The Effect of Residual Palladium Catalyst Contamination on the Photocatalytic Hydrogen Evolution Activity of Conjugated Polymers. Advanced Energy Materials 2018, 8, 1802181.

40. Sachs, M.; Cha, H.; Kosco, J.; Aitchison, C. M.; Francàs, L.; Corby, S.; Chiang, C.-L.; Wilson, A. A.; Godin, R.; Fahey-Williams, A.; Cooper, A. I.; Sprick, R. S.; McCulloch, I.; Durrant, J. R., Tracking Charge Transfer to Residual Metal Clusters in Conjugated Polymers for Photocatalytic Hydrogen Evolution. Journal of the American Chemical Society 2020, 142, 14574-14587. 
41. Prentice, Andrew W.; Zwijnenburg, M. A., Hydrogen Evolution by Polymer Photocatalysts; a Possible Photocatalytic Cycle. Sustainable Energy \& Fuels 2021, 5, 26222632.

42. Sprick, R. S.; Aitchison, Catherine M.; Berardo, E.; Turcani, L.; Wilbraham, L.; Alston, B. M.; Jelfs, K. E.; Zwijnenburg, M. A.; Cooper, A. I., Maximising the Hydrogen Evolution Activity in Organic Photocatalysts by Co-Polymerisation. Journal of Materials Chemistry $A$ 2018, 6, 11994-12003.

43. Kosco, J.; Bidwell, M.; Cha, H.; Martin, T.; Howells, C. T.; Sachs, M.; Anjum, D. H.; Gonzalez Lopez, S.; Zou, L.; Wadsworth, A.; Zhang, W.; Zhang, L.; Tellam, J.; Sougrat, R.; Laquai, F.; DeLongchamp, D. M.; Durrant, J. R.; McCulloch, I., Enhanced Photocatalytic Hydrogen Evolution from Organic Semiconductor Heterojunction Nanoparticles. Nature Materials 2020, 19, 559-565.

44. Sachs, M.; Sprick, R. S.; Pearce, D.; Hillman, S. A. J.; Monti, A.; Guilbert, A. A. Y.; Brownbill, N. J.; Dimitrov, S.; Shi, X.; Blanc, F.; Zwijnenburg, M. A.; Nelson, J.; Durrant, J. R.; Cooper, A. I., Understanding Structure-Activity Relationships in Linear Polymer Photocatalysts for Hydrogen Evolution. Nature Communications 2018, 9, 4968.

45. Sprick, R. S.; Bonillo, B.; Clowes, R.; Guiglion, P.; Brownbill, N. J.; Slater, B. J.; Blanc, F.; Zwijnenburg, M. A.; Adams, D. J.; Cooper, A. I., Visible-Light-Driven Hydrogen Evolution Using Planarized Conjugated Polymer Photocatalysts. Angewandte Chemie International Edition 2016, 55, 1792-1796.

46. Sekar, A.; Sivula, K., Organic Semiconductors as Photoanodes for Solar-Driven Photoelectrochemical Fuel Production. CHIMIA International Journal for Chemistry 2021, 75, 169-179.

47. Wang, Q.; Domen, K., Particulate Photocatalysts for Light-Driven Water Splitting: Mechanisms, Challenges, and Design Strategies. Chemical Reviews 2020, 120, 919-985.

48. Chen, S.; Takata, T.; Domen, K., Particulate Photocatalysts for Overall Water Splitting. Nature Reviews Materials 2017, 2, 17050. 